\title{
Granulocyte chemotaxis: multiple assay screening using a raft technique
}

\author{
U JAYASWAL, S ROPER, S ROATH
}

From the Department of Haematology, Faculty of Medicine, Southampton General Hospital, Southampton SO9 $4 X Y$

SUMMARY The assessment of granulocyte chemotaxis is complicated by the difficulty of precisely $\dot{\omega}_{\pi}$ reproducing results in serial estimations and deciding on the best end point which would reflect most iv accurately the degree of travel taken by the cells under observation. The methods in use are generally based on the Boyden chamber ;" following this, we have further developed the principle of the "raft" technique of chamber based migration. ${ }^{2}$ In order to overcome the problems associated with reproducibility of results when performing multiple assays of chemotaxis, especially when sera of widely differing activity are encountered in the screening procedure, we have used a "batching", system and a simple method of presenting the results so that they are comparable.

The means of quantifying the degree of granulocyte migration most commonly employed is the "leading front" method; 3 we have found this unsatisfactory even at $90 \mathrm{~min}$ for faster-moving granulocytes or stronger chemoattractants, as some of the cells move beyond the membrane and may be lost to assay. ${ }^{34}$ In these circumstances, three possible solutions exist:

(i) the micropore membrane could be thickermany authors ${ }^{56}$ say they have been unable to find membranes of standardised thickness and quality and until manufacturers can correct this and provide thicker membranes this solution remains theoretical.

(ii) use a weaker chemotaxin; sometimes assays are done for the express purpose of looking for decreased migration-for example, when an inhibitor is present; in such cases designated chemotaxins need to be used against the special nature of the inhibitor-for example, C5a for C5a specific inhibitor. ${ }^{7} 8$ Thus the second solution has limited application.

(iii) a shortening of incubation time from $3 \mathrm{~h}$ to around 60 to 90 min has already been employed by all the investigators using the "leading front" estimation.

However, if one is looking for inhibitors, then the chemotaxin should give relatively long leading fronts so that shortening can be more clearly observed. In our work we regularly use an attractant which produced a leading front of at least $100 \mu \mathrm{m}$ in $90 \mathrm{~min}$.

Some of the difficulties were overcome by Maderazo and Woronick ${ }^{5}$ who described a leucotactic index (LI) which required that the incubation

Accepted for publication 17 June 1981 time be flexible between 60 and $90 \mathrm{~min}$ to prevent the leading front from penetrating the whole of the permeable membrane (see later). We describe a method of assessment using a uniform incubation time which appears to retain the sensitivity of the LI but requires no time adjustments and a simple apparatus. It is best suited for multiple assays especially where there is a wide range of chemotactic activity in the sera to be examined-especially on a serum prior to further evaluation depending on the results obtained. This method of recording and presenting results also illustrates the pattern of migration.

\section{Material and methods}

Normal human group $O$ granulocytes from dextran sedimentation of whole blood are washed twice in Hanks basal salt solution (HBSS) and the cell button is then resuspended in the same with $0.2 \%$ human serum albumin (HSA) added. The final cell suspension contains about $4 \times 10^{9}$ leucocytes/l.

Pooled fresh frozen $A B$ serum $(P A B)$ from glassclotted whole blood (stored in aliquots at $-70^{\circ} \mathrm{C}$ until required) is the positive control and standard stimulant when used as $10 \% / 20 \%$ dilution in HBSS + HSA. Other sera were also tested.

The "raft plate" of thick, clear polished perspex $300 \mathrm{~mm} \times 150 \mathrm{~mm} \times 5 \mathrm{~mm}$ is divided into 36 equal islands of $48 \mathrm{~mm} \times 22 \mathrm{~mm}$ area by grooves of $2.5 \mathrm{~mm} \times 2 \mathrm{~mm}$. This is readily made and suitable 182 
for 36 duplicate/triplicate tests. A ventilated lid protects the rafts (Figs. 1 and 2). We find that the rafts do not need to be in containers but each set of replicates rests on its own island of saturated membrane which is of a standard area and keeps the liquid attractant in situ by surface tension.

The cell chambers are $8 \mathrm{~mm}$ diameter plastic caps (LP/35, Luckham Ltd, Sussex) which hold about $250 \mu \mathrm{l}$ of cell suspension each.

Micropore membranes $(3 \cdot 0 \mu \mathrm{m})$ (SSWPO/90R, Millipore Corp, Massachusetts) were used for the screening tests.

Reservoirs for the stimulant consist of blank, clean antibiotic assay pads (Whatman, England) of $13 \mathrm{~mm}$ diameter capable of holding $200 \mu \mathrm{l}$ of fluid at least.

The tests require a little dexterity to set up as the discs are placed on the islands in replicates touching each other, then the membranes are placed over the discs, and the whole saturated from below with

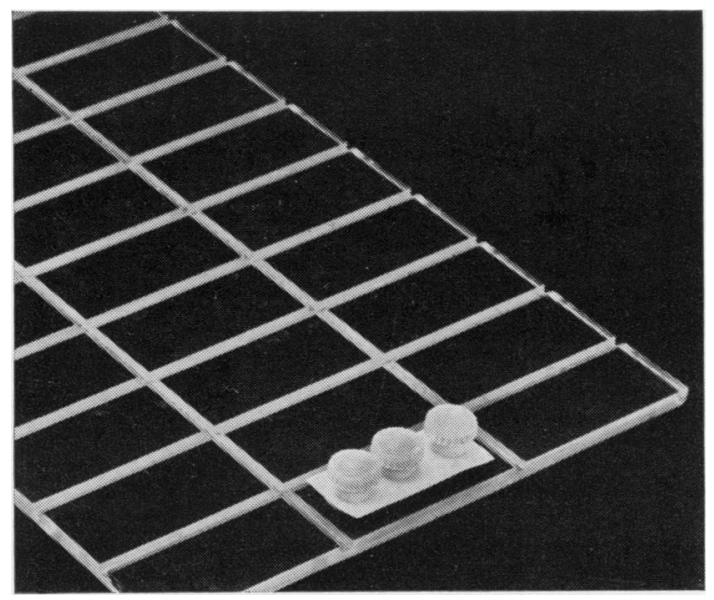

Fig. 1 Part of the chemotaxis plate with a triplicated "raft".

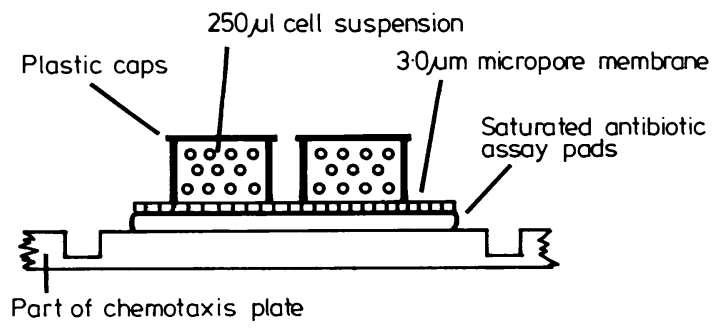

Fig. 2 Modified raft technique. stimulant fluid, $250 \mu \mathrm{l}$ per replicate. We normally use duplicates and so one third of the membrane is cut off and discarded. Complete wetting of the membranes and discs is mandatory. The caps containing neutrophils are inverted over the appropriate membrane and then slightly tapped to ensure that there is no air lock and the plate covered by a lid and placed in a $\mathrm{CO}_{2}$ enriched, moist incubator at $37^{\circ} \mathrm{C}$ for $90 \mathrm{~min}$. After incubation is complete, the plate is removed from the incubator, the caps are removed gently but rapidly, the membranes are transferred to a similar old and used plate (kept for staining only) flooded with absolute alcohol to fix the cells and then washed, stained with haematoxylin, thoroughly dehydrated with ethanol and rinsed with propanol. When dry (be careful not to distort them), the membranes are cleared on a glass slide with xylol and then mounted on the slide with the distal surfaces uppermost. Up to 36 sets of replicates can be assessed during one "run" using the plate described.

Measurement of migration is achieved by viewing the membranes under the $\times 40$ objective lens of a microscope fitted with a micrometer so that vertical travel of the objective lens can be recorded. If complete penetration is present, the degree of migration is assessed by the following means:

The number of cells per high power field is counted at five levels of successive $20 \mu \mathrm{m}$ distances from the distal (upper) surface of the membrane. This is repeated at three sites on the membrane and the average number is noted and fed into a computer which is programmed to calculate the percentage of the cumulative sum of cells that would have actually passed each $20 \mu \mathrm{m}$ level. From the formula $\mathrm{y}=\mathrm{mx}$ $+\mathrm{c}$

$y=\%$ cumulative sum of the migrating cells. $\mathrm{x}=$ the depth $(\mu \mathrm{m})$ into the membrane of each place where cells were counted, and

$\mathrm{c}=$ constant.

$\mathrm{m}=$ the gradient of migration and can be calculated. (Fig. 3 shows examples of appropriate readings.)

Any programmable calculator or small table computer can be programmed to handle the raw data and produce, among other derivatives, the value of $m$ for each curve, thereby enabling a meaningful comparison between controls and tests to be made. We used the Casio FX-502P calculator or CBM Commodore PET computer with the standard least squares fit for a straight line programme - that is, the above formula, when there was complete penetration of the micropore membrane.

\section{Results}

The Table shows how the migration gradient is 
Examples of calculating the percentage cumulative sum from raw data. (Four sets of averaged cell count/high power field using normal group $O$ neutrophils migrating towards $10 \%$ PAB.HSA)

\begin{tabular}{|c|c|c|c|c|c|c|c|c|}
\hline \multirow{3}{*}{$\begin{array}{l}\text { Depth }(\mu m) \text { where cells were } \\
\text { counted }\end{array}$} & \multicolumn{8}{|l|}{ Volunteers } \\
\hline & \multicolumn{2}{|l|}{$R M$} & \multicolumn{2}{|l|}{$U J$} & \multicolumn{2}{|l|}{$E S$} & \multicolumn{2}{|l|}{$S W$} \\
\hline & Cell count & {$[\%]$} & Cell count & {$[\%]$} & Cell count & {$[\%]$} & Cell count & {$[\%]$} \\
\hline 20 & 33 & 100 & 38 & 100 & 39 & 100 & 39 & 100 \\
\hline 40 & 32 & 83 & 35 & 82 & 27 & 79 & 27 & 64 \\
\hline 60 & 32 & 67 & 34 & 66 & 30 & 64 & 18 & 42 \\
\hline 80 & 29 & 51 & 32 & 51 & 32 & 48 & 13 & 22 \\
\hline 100 lower surface & $33 \div 38$ & 36 & $46 \cdots 32$ & 36 & 34 & 31 & $11 \cdot 0$ & 10 \\
\hline Total cells & 197 & 100 & 217 & 100 & 190 & 100 & 108 & 100 \\
\hline $\mathrm{m}$ value & \multicolumn{2}{|c|}{-0.80} & \multicolumn{2}{|c|}{-0.80} & \multicolumn{2}{|c|}{-0.84} & \multicolumn{2}{|c|}{$-1 \cdot 11$} \\
\hline
\end{tabular}

$[\%]=\%$ of the cumulative sum.

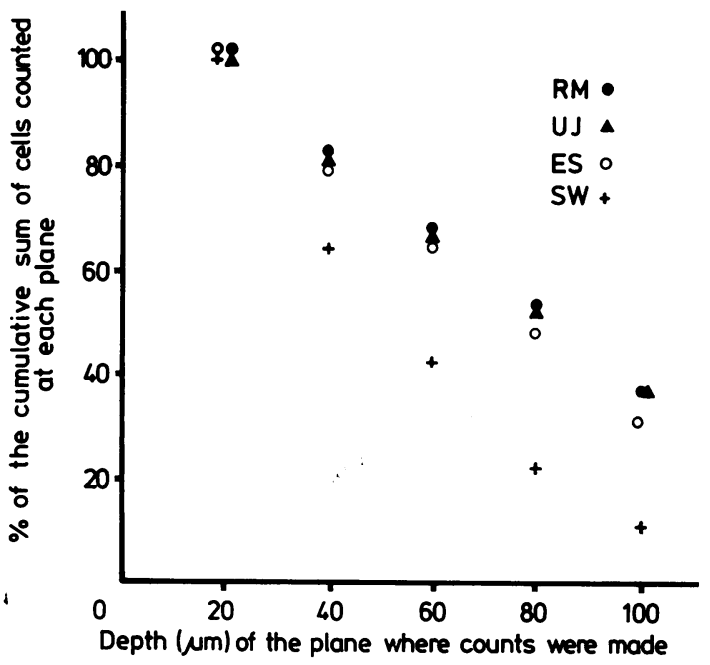

Fig. 3 Percentage cumulative sum values plotted against the planes where cells were counted. (The gradient of the best straight line drawn through the points is the migration gradient or $m$ value of that test).

obtained. In Fig. 4 the migration gradients (m values) of tests (from many separate days) are plotted against their calculated leucotactic index (LI), using counts at every $20 \mu \mathrm{m}$ rather than $10 \mu \mathrm{m}$ between each plane. All completely penetrating cells are assumed to have traversed $120 \mu \mathrm{m}$, although many would have actually moved further. It can be seen in Fig. 4 that there is a correlation between $\mathrm{LI}$ and $\mathrm{m}$ value, albeit a biphasic one where there is a steep deviation from the initial incline when LI $>60$. This is to be expected as there is undervaluation of the LI when the speed of the fastest-moving cells are all approximated to $120 \mu \mathrm{m}$. The $m$ value looks at the whole population and the speed of the majority of the cells (the mode) and so those fastest cells would not be causing errors when the $\mathrm{m}$ value is calculated. These data confirm potential value of $\mathrm{m}$ gradient in a situation where

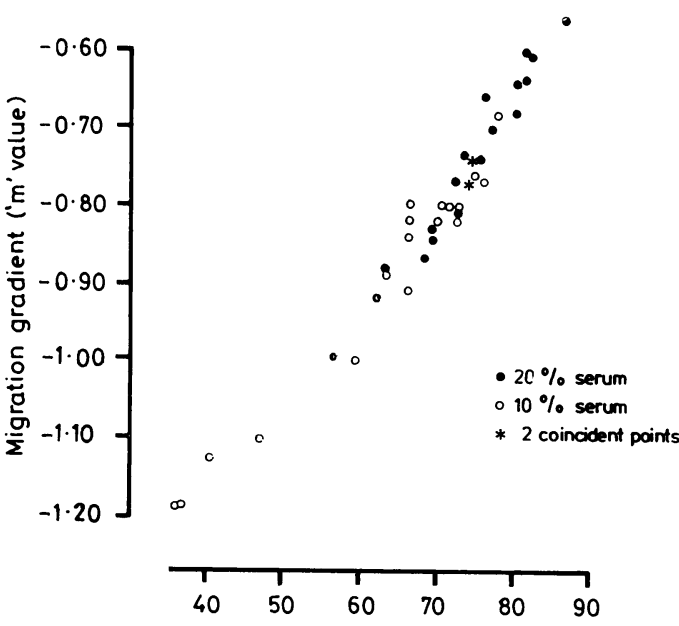

Fig. 4 Correlation of $m$ value to leucotactic index $(L I)$.

considerable cell movement is occurring-for example, in the presence of strong in vitro chemotaxins. Most chemotaxis measurements using the Boyden chamber are fairly time-consuming, but using the raft modification a larger number of tests may be completed in a short time, due to the reduction in incubation to $90 \mathrm{~min}$. (An even shorter time is possible, but then only the fastest-moving cells-the leading front-and not the whole population are measured.)

However using the $90 \mathrm{~min}$ incubation time as a standard, only materials showing marked inhibition of chemotaxis would be detected by the leading front method. Enhancing or lessening of inhibitory activity, would not be detected because of the inadequate thickness of the membranes (the cells having passed through them). Although Maderazo and Woronick ${ }^{5}$ have shown that the LI is a good measure of the performance of the whole cell 
population, their method required alteration of incubation time to suit each test, so that regardless of activity of cells and serum, incomplete penetration of the membrane must be obtained.*

We believe this makes for poor comparison in many cases by introducing an unnecessary variable into the test. Although there are obviously cells that move at different speeds, nonetheless as many measurement conditions should be as standardised as possible. We suggest that the measurement of the migration gradient or $\mathrm{m}$ value is a better method of assessing average cell movement and this method takes into account any variation in the speed of the granulocyte populations. Most importantly, this method gives a dynamic picture of what is actually happening and given the depths of the planes where cells were counted and the $\mathrm{m}$ value, the actual migration curve could easily be reconstructed (conveniently by computer) if necessary.

We find pooled $\mathrm{AB}$ serum a potent chemotaxin but can only compare the attractant activity of these sera and also normal sera of various blood groups using their migration gradients; neither the leading front nor the LI could be accurately calculated from the figures obtained after the $90 \mathrm{~min}$ incubation. For known slow-moving cells or weak attractants the LI or even the leading front estimation can be of course used as the simple method of reporting chemotaxis, and we continue to use the latter for convenience when comparing such materials.

The data suggest that our method is more useful than the LI and more easily standardised and it is

*The migrated cells were counted at $10 \mu \mathrm{m}$ intervals from the original monolayer on the cell surface. The number of cells per high power field at each level was multiplied by the distance of that level from the starting surface. The products were added and the sum was then divided by the total number of cells at all layers counted per high power field. The results make up the LI for that incubation time. eminently suitable for looking at the pattern of migration as opposed to the average speed of a cell whatever the speed of migration. We confirm that the raft technique is well suited for routine multiple screening, especially if performed as described here.

We gratefully acknowledge receipt of a grant from the Leukaemia Research Fund (No. 78/21).

We are also grateful to Dr F Paul for help in the developing of the PET programme used for our calculations of migration gradient ( $m$ value).

\section{References}

${ }^{1}$ Boyden S. The chemotactic effect of mixtures of antibody and antigen on polymorphonuclear leucocytes. $J$ Exp Med 1962;115:453-66.

${ }^{2}$ Addison IE, Babbage JW. A raft technique for chemotaxis: A versatile method suitable for clinical studies. $J$ Immunol Methods 1976;10:385-8.

${ }^{3}$ Zigmond SH, Hirsch JG. Leucocyte locomotion and chemotaxis. J Exp Med 1973;137:387-410.

${ }^{4}$ Keller HU, Gerber H, Hess MW, Cottier H. Studies on the regulation of the neutrophil chemotactic response using a rapid and reliable method for measuring random migration and chemotaxis of neutrophil granulocytes. Agents Actions 1976;6:326-38.

${ }^{5}$ Maderazo EG, Woronick CL. Micropore filter assay of human granulocyte locomotion: problems and solutions. Clin Immunol Immunopathol 1978;11:196-211.

${ }^{6}$ Keller HU, Sorkin E. Studies on chemotaxis IX. Migration of rabbit leucocytes through filter membranes. Proc Soc Exp Biol Med 1967;126:677-80.

${ }^{7}$ Wright DG, Gallin JI. Mechanisms for modulation of the inflammatory response: generation and inactivation of C5a by products stored in granules of human neutrophils. In: Gallin JI, Quie PG, eds. Leucocyte chemotaxis. New York: Raven Press, 1978.

${ }^{8}$ Goetzl EJ, Austen KF. A neutrophil immobilizing factor derived from human leucocytes. 1 . Generation and partial characterization. J Exp Med 1972;136:1564-80.

Requests for reprints to: Dr U Jayaswal, Faculty of Medicine, Southampton General Hospital, Tremona Road, Southampton SO9 4XY. 